\title{
Spectrum Handoff Decisions
}

\author{
Bisma Bashir ${ }^{1}$, Er. Tajinder Pal Singh ${ }^{2}$ \\ ${ }^{1} \mathrm{M}$. Tech Scholar, ${ }^{2}$ Assistant Professor \\ Department of Electronics and Communication Engineering, \\ Sri Sai College of Engineering \& Technology Badhani, Pathankot, Punjab, India
}

\begin{abstract}
In modern society wireless communication plays a very important role with huge increase of users, services and standards. Although there are several pros of using cognitive radio networks, for example increasing the spectrum utilization of wireless networks, but still there exist some challenges which need to be discussed in detail: First, since cognitive radio networks are planned to operate over a heterogeneous spectrum environment, which comprises of both licensed and unlicensed spectrum bands, most of the research has been done in only the licensed band of the spectrum and only some research has been carried out in such an environment as merging licensed and unlicensed spectrum bands for transmission increases the spectrum utilization of cognitive radio wireless networks. There is need to develop new spectrum handoff schemes to improve the performance of the secondary users in terms of their handoff delay.
\end{abstract}

\section{KEYWORD: CR, SDR, DSA.}

\section{INTRODUCTION}

With most of our activities implying some kind of wireless access in one way or other, wireless communication has now become extremely important in modern society. As a result, applications and services, standards, and the number of wireless users, are increasing day by day, considering that most of the limited available radio spectrum resources have been allocated well and can restrict this growth. Also, this amount of expansion is ultimately controlled by the available radio frequency spectrum; government regulatory agencies have adopted, until recently, a strict approach to the licensing of limited amounts of spectrum to different entities (e.g., military, analogue cellular telephony, service providers, public safety and TV), all of these entities possess exclusive transmissions to their assigned frequency channels. By using this strict approach, the main method of accessing a radio spectrum resource is based on a fixed spectrum allocation method, known as Fixed Spectrum Access (FSA). A report prepared by the Spectrum Policy Task Force (SPTF) and published by The Federal Communication Commission (FCC), which gave a proof to the fact that most of the dedicated (licensed) spectrum experiences low utilization efficiency, such as those allocated to a TV or analogue cellular telephony, which are not fully utilized. The study revealed both temporal and geographic changes in spectrum utilization, ranging from $15-85 \%$ in the bands below $3 \mathrm{GHz}$, while ranging lower than this at higher frequencies. These temporally unused spectrums, called spectrum holes or white spaces, are illustrated further in following figure. These spectrum holes are sometimes known as the "virtual channels". These virtual channels are logical channels built over the spectrum holes of the licensed PU channels.

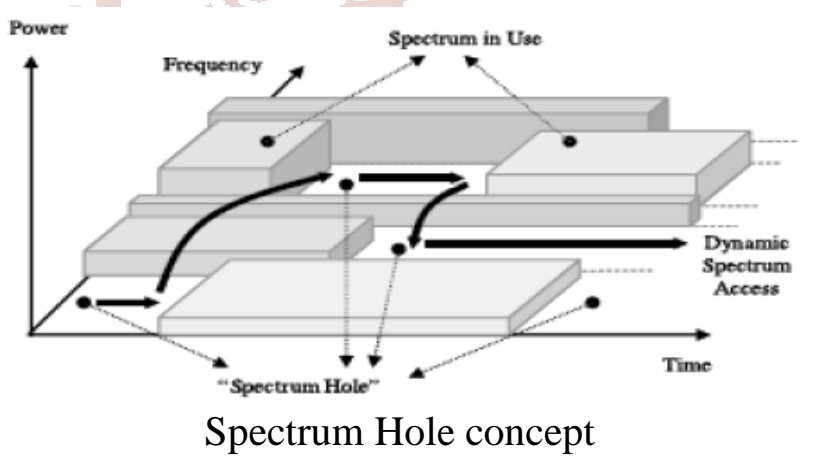

\section{RADIO SPECTRUM \& COGNITIVE RADIO NETWORKS}

In most of the countries of the world, the precious radio spectrum is controlled by government. The usage of the radio spectrum is controlled by regulatory bodies defined by each country's 
government such as of com in the UK and FCC in the USA; enforce the spectrum allocation paradigm "command-and-control". The legacy of fixed spectrum access (FSA) policy is followed by almost all existing wireless devices and networks in order to use the radio spectrum, in which the spectrum bands are licensed to being devoted (primary) services and users, such as cellular networks, TVs, and vehicular ad hoc networks but only the primary users can operate in the assigned spectrum in these systems. On the other hand, the other systems are not allowed to use this spectrum, even when it is idle. Although the issue of interference among different devices and networks can be efficiently controlled and coordinated by using FSA, this policy still causes significant spectrum under-utilization, as reported in. The growth of new wireless services and applications along with diverse network architectures (such as heterogeneous networks) has been adversely affected by the inefficiency of the radio spectrum. As a consequence, new flexible and dynamic spectrum regulations should be introduced in order to overcome this issue.

\section{COGNITIVE RADIO}

The exploration of the cognitive radio trend involves an interdisciplinary effort from different technical areas, including: communications engineering, the networking, spectrum policies, adaptive systems and learning, signal processing, the information theory, the game/cooperative theory, economics and social sciences.

Although there have been many advances in the cognitive radio trend with respect to enabling DSA networks, further study is required to make DSA a feasible spectrum allocation paradigm in CRNs. Major existing studies on cognitive radios cover:

1. Spectrum policy alternatives and system models

2. Spectrum sensing algorithms

3. Cognitive radio architecture and software abstractions

4. Cognitive algorithms for adaptation and resource management

5. DSA technology and algorithms

6. Protocol architectures for CRNs

7. Network security for CRNs

8. Cooperative wireless communications

9. Cognitive medium access control (MAC)

10. Cognitive networking and the Internet

11. Game theory for cognitive radio networks

12. Physical layer aspects
Some of research focuses on the implementation of CRs which require no changes in existing networks (infrastructures). Other research suggests more than a single radio for each secondary user, or they assume secondary users have a wide band spectrum sensing. The following subsections introduce some basic and vital aspects and definitions related to the cognitive radio.

\section{SOFTWARE-DEFINED RADIO (SDR)}

The rapid evolution of microelectronics has enabled the development of software-defined-radio (SDR) technology, where the baseband digital processing is completely achieved in software. This technology allows to adapt to operating parameters (e.g., carrier frequency, transmit power, coding scheme and modulation strategy), based on learning from previous events and current inputs to the system. This gives opportunities to unlicensed users to access the licensed spectrum without interfering with the primary users.

\section{CHALLENGES OF COGNITIVE RADIO} TECHNOLOGY

The two main aspects of cognitive radio are:

1. Cognitive /Capability: Because of the high variation in the available spectrum and

2. The diversity of applications that can be used by CR technology, CR users must be able to:

Identify and detect which parts of the spectrum are available at a specific time and location through real time interaction with the surrounding environment (spectrum wideband sensing)

Choose the appropriate band for transmission (spectrum decision)

Share access processing with other CR users (spectrum sharing)

Vacate the spectrum when a primary user appears in the selected band (spectrum mobility)

These network capabilities can be realized by spectrum management functions (see Section 2.9).

Re-configurability: CR users must have the following abilities:

They must be able to adapt to the modulation scheme in order to improve spectrum access process.

$>$ They must be able to operate on different systems with different protocols in a wideband frequency range supported by its hardware design. Using this capability, the best networks and channels can be 
selected and then CR users are able to reconfigure themselves to be compatible with the new environment.

They must be able to frequency agility, which means the ability of the CR user can change its transmission frequency.

$>$ They must be able to control the transmission power, for example, transmitting power control can be used to control the power level dynamically, which reduces the emitted power to allow greater sharing of the spectrum when a higher power level is not necessary.

\section{CONCLUSION}

The research paper describes the spectrum handoff decisions. The paper introduces Cognitive Radio with full information of Software Defined Radio (SDR). The paper also describes the Challenges of Cognitive Radio Technology. This paper addresses one of the fundamental problems of signal drops during traversing through heterogeneous network. The method of cognition allows the best spectrum selection ensuring lossless handoffs.

\section{REFERENCES:}

1. Wyglinski and M. Nekovee, "Cognitive radio communications and networks," IEEE Communications Magazine, vol. 46, pp. 30-31, 2008.

2. E. FCC, "Docket No 02-135," Spectrum Policy Task Force Report, 2002.

3. S. M. Mishra, D. Cabric, C. Chang, D. Willkomm, B. Van Schewick, S. Wolisz, and B. Brodersen, "A real time cognitive radio test bed for physical and link layer experiments," 2005, pp. 562-567.

4. S. Huang, X. Liu, and Z. Ding, "Optimal transmission strategies for dynamic spectrum access in cognitive radio networks," Mobile Computing, IEEE Transactions on, vol. 8, pp. 1636-1648, 2009.

5. F. Akyildiz, W. Y. Lee, M. C. Vuran, and S. Mohanty, "A survey on spectrum management in cognitive radio networks," Communications Magazine, IEEE, vol. 46, pp. 40-48, 2008.

6. S. Haykin, "Cognitive radio: brain-empowered wireless communications, "Selected Areas in Communications, IEEE Journal on, vol. 23, pp. 201-220, 2005.

7. A. Fette, Cognitive radio technology: Academic Press, 2009.

8. L. Kleinrock, "Queueing Systems, Vol. II: Computer Applications," ed:Wiley, New York, 1976.

9. L. Kleinrock, "Queueing systems, vol. I: Theory," ed: Wiley, New York, 1975.

10. Available:

http://www.cse.fau.edu/ bob/publications/encyclo pedia.pdf

11. J. Walraevens, D. Fiems, and H. Bruneel, "Performance analysis of priorityqueueing systems in discrete time," in Network performance engineering, ed: Springer, 2011, pp. 203-232.

12. M. Tanner, Practical Queueing Analysis: McGraw-Hill, 1995.

13. Efrosinin and A. Winkler, "Queueing system with a constant retrial rate, non-reliable server and threshold-based recovery," European journal of operational research, vol. 210, pp. 594-605, 2011.

14. B. Choi and Y. Chang, "Single server retrial queues with priority calls, "Mathematical and Computer modelling, vol. 30, pp. 7-32, 1999. 